\title{
Edoxaban schützt auch vor dem zweiten ischämischen zerebralen Ereignis
}

Fragestellung: Wie sicher und wirksam ist der Faktor-Xa-Hemmer Edoxaban in der Sekundärprävention des Schlaganfalls bei Patienten mit Vorhofflimmern?

Hintergrund: Patienten mit Vorhofflimmern, die eine transiente ischämische Attacke (TIA) oder einen Schlaganfall erlitten haben, weisen ein besonders hohes Rezidivrisiko auf. Deshalb ist bei ihnen eine orale Antikoagulation noch wichtiger als in der Primärprävention. In den Studien, in denen die Nicht-Vitamin-K-abhängigen oralen Antikoagulanzien (NOAK) Apixaban, Dabigatran und Rivaroxaban im Vergleich zu Warfarin untersucht worden waren, sind die Ergebnisse der Untergruppen in der Sekundärprävention publiziert [1]. Metaanalytisch führen diese drei NOAK zu einer signifikanten Reduktion erneuter ischämischer Schlaganfälle und insbesondere zu einer dramatischen Reduktion von intrakraniellen Blutungen. Die Ergebnisse für Edoxaban waren bisher nicht publiziert worden.

Patienten und Methodik: Die Studie ENGAGE AF-TIMI 48 randomisierte 21.105 Patienten mit Vorhofflimmern, die entweder Warfarin oder Edoxaban (einmal täglich $30 \mathrm{mg}$ oder $60 \mathrm{mg}$ ) erhielten. Die mittlere Beobachtungszeit betrug 2,8 Jahre. Die primären Endpunkte waren Schlaganfall, systemische Embolie und schwerwiegende Blutungskomplikationen.

Rost NS, Giugliano RP, Ruff CT et al. Outcomes with edoxaban versus warfarin in patients with previous cerebrovascular events: Findings from ENGAGE AF-TIMI 48 (Effective Anticoagulation With Factor Xa Next Generation in Atrial Fibrillation-Thrombolysis in Myocardial Infarction 48). Stroke 2016; 47: 2075-82
Ergebnisse: Im Rahmen der Studie hatten 5.973 Patienten (28,3\%) bereits eine zerebrale Ischämie oder eine TIA erlitten. $36 \%$ der Patienten waren über 75 Jahre alt. Patienten mit vorausgegangener TIA oder ischämischem Insult hatten verglichen mit den $\mathrm{Pa}$ tienten ohne ein solches Ereignis in der Anamnese ein

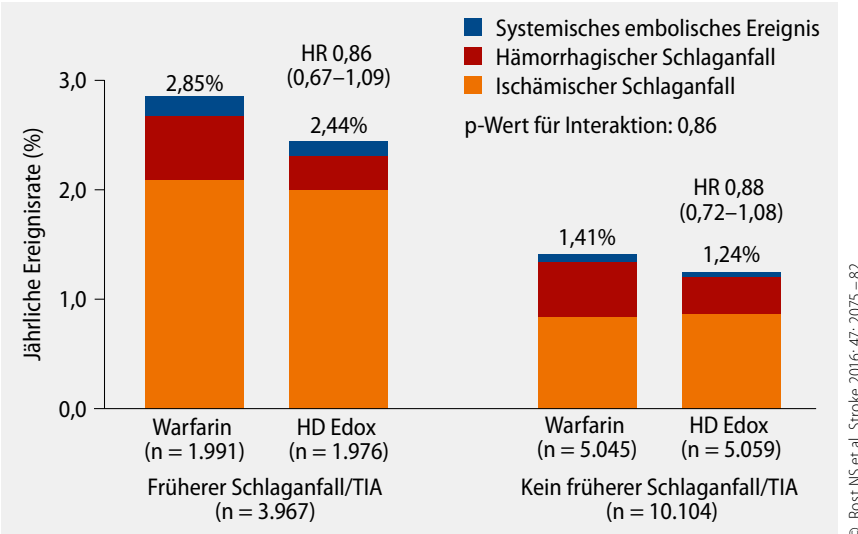

1 Primärer Wirksamkeitsendpunkt unter Edoxaban 60 mg/Tag.

signifikant erhöhtes Risiko thromboembolischer Ereignisse (2,83\% vs. $1,42 \%$ pro Jahr). Dasselbe galt für schwerwiegende Blutungskomplikationen (3,03\% vs. 2,64\% pro Jahr).

Die Rate an Schlaganfällen und systemischen Embolien betrug $2,85 \%$ pro Jahr in der Warfarin-Gruppe und 2,44\% für die mit der höheren Edoxaban-Dosis behandelten Patienten. Dies entspricht einer Hazard Ratio (HR) von 0,86, die nicht signifikant war. Für intrakranielle Blutungen betrug die Rate unter Warfarin $1,09 \%$ pro Jahr und unter der höheren Edoxaban-Dosis 0,62 \% pro Jahr. Dieser Unterschied war mit einer HR von 0,57 signifikant.

Schlussfolgerungen: Edoxaban ist in der Sekundärprävention thromboembolischer Ereignisse bei Patienten mit Vorhofflimmern genauso wirksam wie in der Primärprävention verglichen mit dem Vitamin-K-Agonisten Warfarin. Sowohl in der Primärals auch in der Sekundärprävention ist das Risiko von schwerwiegenden Blutungskomplikationen und intrakraniellen Blutungen unter Edoxaban niedriger als unter Warfarin.

\section{- Kommentar von Hans-Christoph Diener, Essen}

\section{Vergleichbare Ergebnisse wie mit den anderen NOAK}

Die Studie ENGAGE AF-TIMI 48, die Edoxaban in der Schlaganfallprävention bei Vorhofflimmern untersuchte, hat im Prinzip dasselbe Resultat für die Sekundärprävention wie die drei Studien mit den anderen NOAK gezeigt. Generell reduzieren NOAK tendenziell das Risiko erneuter Schlaganfälle, wobei die jeweiligen Gruppengrößen in den einzelnen Studien nicht groß genug waren, um einen signifikanten Unterschied zu erzielen. Ähnlich wie in den mit Apixaban, Dabigatran und Rivaroxaban durchgeführten Studien zeigte sich auch für den Fak-
tor-Xa-Hemmer Edoxaban ein starker Trend für geringere Raten an schwerwiegenden Blutungskomplikationen und eine signifikante Reduktion des Risikos intrakranieller Blutungen. Eine neue Metaanalyse, welche die Ergebnisse aller vier Studien in der Sekundärprävention bei Patienten mit Vorhofflimmern untersucht, liegt allerdings noch nicht vor.

\footnotetext{
Referenz:

1. Ntaios $\mathrm{G}$ et al. Stroke 2012; 43: $3298-304$
} 